\title{
EFECTO DE LA FERTILIZACIÓN ORGÁNICA Y QUÍMICA EN EL RENDIMIENTO DE FRUTO DE CHILE POBLANO
}

\section{EFFECT OF ORGANIC AND CHEMICAL FERTILIZATION ON FRUIT YIELD OF POBLANO PEPPER}

\author{
Ana M. Tlelo-Cuautle, Oswaldo R. Taboada-Gaytán*, Javier Cruz-Hernández, \\ Higinio López-Sánchez y Pedro A. López
}

\begin{abstract}
Colegio de Postgraduados, Campus Puebla, Programa en Estrategias para el Desarrollo Agrícola Regional, Santiago Momoxpan, Municipio de San
\end{abstract} Pedro Cholula, Puebla, México.

*Autor de correspondencia (toswaldo@colpos.mx)

\section{RESUMEN}

El uso de fertilizantes químicos y estiércoles es una práctica común entre los agricultores que cultivan chile Poblano (Capsicum annuum L. cv. Poblano), pero no se han realizado estudios experimentales para definir la mejor combinación para aumentar el rendimiento de fruto en este cultivo. El objetivo del presente estudio fue evaluar el efecto de tres fórmulas de fertilización química (120N-60P-120K, 80N-40P-80K y 40N-20P-40K) combinadas con tres tipos de abonos orgánicos (abono orgánico comercial Solep ${ }^{\circledR}$ y estiércoles locales de ganado ovino y vacuno, aplicados en dosis de 5,10 y $15 \mathrm{t} \mathrm{ha}^{-1}$ ) sobre el rendimiento de fruto de chile poblano. Se utilizó un diseño en bloques completos al azar con dos repeticiones. La combinación de estiércol ovino a una dosis de $15 \mathrm{t} \mathrm{ha}^{-1}$ más fertilizante químico con la fórmula $80 \mathrm{~N}-40 \mathrm{P}-80 \mathrm{~K}$ generó mayor rendimiento $\left(35.5 \mathrm{t} \mathrm{ha}^{-1}\right.$ de fruto verde), altura de planta $(74 \mathrm{~cm})$, número de frutos por planta (22 frutos) y peso de fruto fresco $(86.52 \mathrm{~g})$, mientras que el fertilizante orgánico comercial Solep ${ }^{\circledR}$ a $5 \mathrm{t} \mathrm{ha}^{-1}$ más $^{-1}$ la fórmula $80 \mathrm{~N}-40 \mathrm{P}-80 \mathrm{~K}$ presentó el menor rendimiento $\left(14 \mathrm{t} \mathrm{ha}^{-1}\right.$ de fruto fresco), altura de planta $(50 \mathrm{~cm})$, número de frutos por planta (nueve frutos) y menor peso de fruto $(65.13 \mathrm{~g})$. El estiércol de ovino a nivel alto combinado con fertilizantes químicos a nivel medio generó mayor rendimiento de fruto, con efectos significativos sobre la altura de planta, así como en el número y peso de frutos.

Palabras clave: Capsicum annuum, abonos orgánicos, fertilizantes químicos, fruto verde.

\section{SUMMARY}

The use of chemical fertilizers and manures is a common practice among farmers who grow Poblano pepper (Capsicum annuum L. cv. Poblano); however, no experimental studies have determined the best supplemental combination that increases fruit yield in this crop. The objective of this study was to evaluate the effect of three chemical fertilization formulas (120N-60P$120 \mathrm{~K}, 80 \mathrm{~N}-40 \mathrm{P}-80 \mathrm{~K}$, and $40 \mathrm{~N}-20 \mathrm{P}-40 \mathrm{~K}$ ) combined with three types of organic fertilizer (Solep ${ }^{\circledR}$ commercial organic fertilizer, local sheep manure and cattle manure in doses of 5,10 and $15 \mathrm{tha}^{-1}$ ) on the fruit yield of Poblano pepper. A randomized complete blocks design with two replications was used. The combination of ovine manure at a dose of $15 \mathrm{tha}^{-1}$ plus chemical fertilizer with the formula $80 \mathrm{~N}-40 \mathrm{P}-80 \mathrm{~K}$ increased yield $\left(35.5 \mathrm{t} \mathrm{ha}^{-1}\right.$ fresh fruit), plant height $(74 \mathrm{~cm})$, number of fruits per plant (22 fruits), and fresh fruit weight $(86.52 \mathrm{~g})$. The commercial organic fertilizer Solep ${ }^{\circledR}$ at $5 \mathrm{t} \mathrm{ha}^{-1}$ plus the formula $80 \mathrm{~N}-40 \mathrm{P}$ $80 \mathrm{~K}$ reduced yield $\left(14 \mathrm{t} \mathrm{ha}^{-1}\right.$ fresh fruit), plant height $(50 \mathrm{~cm})$, number of fruits per plant (nine fruits), and fruit weight $(65.13 \mathrm{~g})$. A high level of sheep manure combined with medium level of chemical fertilizers increased fruit yield, with significant effects on plant height, as well as on the number and weight of fruits.

Index words: Capsicum annuum, chemical fertilizers, fresh fruit, organic manures

\section{INTRODUCCIÓN}

México no es competitivo a nivel internacional en el cultivo de chile Poblano (Capsicum annuum L. Cv. Poblano), pues cuenta con un rendimiento de chile verde de $20.5 \mathrm{t}$ ha-1 y de chile seco de $1.8 \mathrm{t} \mathrm{ha}^{-1}$ (FAOSTAT, 2017). Una de las opciones para incrementar el rendimiento es mejorar la nutrición del cultivo.

La aplicación de fertilizantes orgánicos y químicos es un componente vital en la agricultura, debido a que aportan a las plantas los nutrientes esenciales para su desarrollo y producción; sin embargo, el aumento de la producción depende en gran medida del tipo de fertilizante que se utiliza para complementar la necesidad de nutrientes en las plantas (Moharana et al., 2017). Aliyu (2000) y Gupta et al. (2017) mencionaron que los fertilizantes químicos por sí solos no pueden mantener altos niveles de productividad; además, su uso genera un aumento en el costo de producción, por lo que los productores incorporan abonos orgánicos al suelo, como una alternativa más económica para nutrir sus cultivos e incrementar la producción.

Adicionalmente, el uso de estiércoles como fuente de nutrientes mantiene la dinámica del suelo, favorece el desarrollo vegetal y la vida macro y microbiana, mejora la estructura del suelo y el desarrollo de raíces (Khandaker et al., 2017; López et al., 2012); además, aportan nutrientes como N, P, K, Ca y Mg, que son liberados lentamente y están disponibles para las plantas por mayor tiempo; también contienen materia orgánica, lo que promueve tanto la 
nutrición de las plantas como el reciclaje de nutrientes en el suelo. Debido a que la liberación de los nutrientes es gradual, éstos pueden ser mejor aprovechados por los cultivos (Chami et al., 2013; Okazaki et al., 2010; Zeidan, 2007); por lo tanto, al aplicar fertilizantes químicos en combinación con abonos orgánicos es posible obtener mayores rendimientos, lo que genera mayores ingresos y márgenes económicos para los productores (Bindra et al., 2014; Rodríguez et al., 2010).

Se han reportado investigaciones sobre el uso de fertilizantes químicos y orgánicos en el cultivo de berenjena (Solanum melongena; Montaño et al., 2009), pimiento (Selvakumar et al., 2018), chile (Rodríguez et al., 2010), cebolla (Allium cepa; Álvarez-Hernández et al., 2011), chile jalapeño (Macías et al., 2012), hierba mora (Solanum nigrum; Bvenura y Afolayan, 2013) y sorgo (Sorghum spp., Kamaei et al., 2019). En el caso de chile poblano se ha investigado le mezcla de fertilizantes químicos con estiércol y abono orgánico en la producción de plántula (Acevedo-Alcalá et al., 2020); sin embargo, no se ha investigado el uso de fertilizantes químicos mezclados con fertilizantes orgánicos o estiércoles específicamente en chile Poblano y en particular en la zona de la Sierra Nevada de Puebla, por lo que se desconoce la mejor combinación para generar los mayores rendimientos de fruto. Por lo anterior, el objetivo de esta investigación fue determinar el efecto de la combinación de tres niveles de tres fertilizantes orgánicos con tres niveles de fertilización química para incrementar el rendimiento de fruto en el cultivo de chile poblano.

\section{MATERIALES Y MÉTODOS}

\section{Sitio experimental}

El experimento se estableció en abril de 2016 en Santa María Zacatepec, municipio de Juan C. Bonilla, Puebla; esta localidad se ubica entre los paralelos $19^{\circ} 06^{\prime}$ y $19^{\circ} 09^{\prime}$ $\mathrm{N}$ y los meridianos $98^{\circ} 18^{\prime}$ y $98^{\circ} 23^{\prime} \mathrm{O}$; la altitud varía entre 2100 y 2300 msnm, predominan los suelos arenosos tipo phaeozem; presenta clima templado subhúmedo y lluvias en verano, con una temperatura media anual de 14 a $16^{\circ} \mathrm{C}$ y una precipitación anual de 900 a 1000 mm (INEGI, 2009).

\section{Material vegetal y fertilizantes}

Se utilizó una variedad criolla local de chile poblano identificada como Colecta 11 proveniente de la localidad de San Felipe Teotlalcingo, Puebla, seleccionada por investigadores del Colegio de Postgraduados Campus Puebla. Se usaron plántulas producidas en charolas de 200 cavidades, con una edad de 51 días después de la siembra y una altura promedio de $12 \mathrm{~cm}$.
Para la fertilización orgánica del cultivo se usó estiércol ovino y vacuno disponibles localmente y colectados de pequeñas unidades de producción ganadera de San Nicolás de los Ranchos, Puebla, y el fertilizante orgánico comercial marca Solep ${ }^{\circledR}$ (abono orgánico comercial de la empresa Soluciones Ecológicas de Puebla, Puebla, México). En muestras compuestas de estos abonos se determinaron los parámetros de $\mathrm{pH}$, conductividad eléctrica (CE), humedad, cenizas, materia orgánica (MO), carbono orgánico $(\mathrm{CO})$, nitrógeno $(\mathrm{N})$, fósforo $(\mathrm{P})$, potasio $(\mathrm{K})$, calcio (Ca) y magnesio $(\mathrm{Mg})$ mediante la metodología establecida en la norma NMX-FF-109-SCFI-2008 de humus de lombriz (Secretaría de Economía, 2008).

\section{Tratamientos}

Se evaluaron tratamientos con aplicación de tres dosis de estiércol ovino y vacuno, así como de fertilizante orgánico comercial Solep ${ }^{\circledR}$ y tres fórmulas químicas (40N-20P-40K, 80N-40P-80K y 120N-60P-120K), así como las combinaciones posibles de ambos (Cuadro 1). Las fuentes utilizadas para la elaboración de las fórmulas químicas fueron la urea (46 \% N), fosfato diamónico (DAP, $18 \% \mathrm{~N}$ y $\left.46 \% \mathrm{P}_{2} \mathrm{O}_{5}\right)$ y el cloruro de potasio $\left(60 \% \mathrm{~K}_{2} \mathrm{O}\right)$. La aplicación de estiércol y la fertilización química se realizó a los 20 y 40 días después del trasplante, respectivamente.

\section{Diseño y unidad experimental}

Para la evaluación de los tratamientos se utilizó un diseño experimental de bloques completos al azar con dos repeticiones. La parcela experimental constó de cuatro surcos de $3.9 \mathrm{~m}$ de largo por $0.8 \mathrm{~m}$ de ancho, con distancia entre plantas de $0.30 \mathrm{~m}$. Cada parcela contenía 48 plantas y se eligieron los dos surcos centrales como parcela útil, para una densidad de población de 38,461 plantas ha-1.

El manejo agronómico se llevó a cabo de acuerdo con las prácticas que tradicionalmente realiza el agricultor de la región en cuanto a preparación del terreno y labores de cultivo.

\section{Variables respuesta}

Se registraron las variables días a floración (DF) y días a fructificación (DFr) en el total de plantas de la parcela útil. Adicionalmente, en una muestra de cinco plantas con competencia completa y representativas fenotípicamente de la parcela útil se determinaron las siguientes variables: altura de planta (AIP) en cm, longitud del tallo (LTa) en cm, diámetro del tallo (DTa) en mm, número de bifurcaciones (NBi), número de frutos (NFr), peso de fruto (PFr) en $g$, longitud del fruto ( $\mathrm{LFr}$ ) en $\mathrm{mm}$, ancho de fruto (AFr) en $\mathrm{mm}$, grosor de fruto (GFr) en $\mathrm{mm}$, espesor del pericarpio 
(EPe) en mm, número de lóculos del fruto (NLF), número de semillas por fruto (NSF), peso de mil semillas (PSe) en g y rendimiento por ha (Ren) en $t \mathrm{ha}^{-1}$. El rendimiento de fruto fresco se determinó al cuantificar el peso promedio de fruto verde por planta, en cinco plantas seleccionadas como representativas de la parcela útil.

\section{Análisis estadístico}

Se realizó un análisis de varianza, prueba de comparación de medias con la diferencia mínima significativa, correlaciones de Pearson y se determinaron contrastes entre los efectos de los abonos orgánicos y las fórmulas de fertilización mediante el programa SAS versión 9.3 (SAS Institute, 2013).

\section{RESULTADOS Y DISCUSIÓN}

El contenido nutricional de los estiércoles y abonos orgánicos se determina a través de las características fisicoquímicas que estos materiales presentan, mismas que son de gran importancia para conocer su calidad nutricional y la materia orgánica que aportan al suelo. El Cuadro 2 muestra las características fisicoquímicas de los estiércoles ovino y vacuno, así como del fertilizante orgánico comercial Solep ${ }^{\circledR}$ utilizados en el estudio.

El estiércol de ovino fue estadísticamente superior $(P \leq 0.001)$ en cuanto a contenido de materia orgánica, carbono orgánico, nitrógeno, magnesio y calcio, por lo que es una buena alternativa para combinarlo con fertilizantes químicos y así complementar los requerimientos nutricionales de las plantas. Una ventaja adicional es que es un material disponible localmente en las pequeñas unidades de producción ganaderas de la región y a un costo mucho menor que el fertilizante orgánico comercial. Aun cuando el contenido de fósforo presenta un valor similar al obtenido en el estiércol vacuno, ambos son estadísticamente diferentes al valor que, para este elemento, presenta el abono comercial.

Se presentaron diferencias estadísticamente significativas $(P \leq 0.05)$ en altura de planta, número de frutos, peso de fruto y rendimiento de fruto por hectárea (Cuadro 3). Se observó aumento en el rendimiento de fruto y mayor altura de planta con la aplicación de fertilizantes químicos en combinación con los estiércoles; estos efectos están asociados con cambios en algunas características morfológicas de la planta, como se ha observado en altura de planta y en longitud y ancho de fruto en chile jalapeño y chile rojo (Ghazali et al., 2016; Macías et al., 2012).

En el Cuadro 4 se observa que con la combinación de estiércol ovino a $15 \mathrm{t} \mathrm{ha}^{-1}$ más la fórmula de fertilización química $80 \mathrm{~N}-40 \mathrm{P}-80 \mathrm{~K}$ se obtuvo el mayor rendimiento de fruto fresco con $35.5 \mathrm{t} \mathrm{ha}^{-1}$, que corresponde al tratamiento 27. El tratamiento que produjo el menor rendimiento fue el 34, que representa la combinación del fertilizante orgánico

Cuadro 1. Relación de tratamientos (TR) del experimento de evaluación de fertilizantes orgánicos y químicos para la producción de fruto de chile poblano.

\begin{tabular}{|c|c|c|c|c|c|}
\hline TR & Descripción & $\mathrm{TR}$ & Descripción & TR & Descripción \\
\hline T1 & 5 tha $^{-1} \mathrm{~V}$ & $\mathrm{~T} 14$ & 10 t ha-1 $^{-1}+120-60-120$ & T27 & 15 t ha-1 $^{-1}+80-40-80$ \\
\hline T2 & $10 \mathrm{t} \mathrm{ha}^{-1} \mathrm{~V}$ & $\mathrm{~T} 15$ & 15 t ha $^{-1} V+120-60-120$ & T28 & 5 t ha-1 $^{-1}+40-20-40$ \\
\hline T3 & 15 tha $^{-1} \mathrm{~V}$ & T16 & 5 t ha $^{-1} V+80-40-80$ & T29 & 10 t ha-1 $^{-1}+40-20-40$ \\
\hline T4 & 5 t ha $^{-1} \mathrm{O}$ & $\mathrm{T} 17$ & 10 tha $^{-1} V+80-40-80$ & Т30 & 15 t ha-1 $^{-1}+40-20-40$ \\
\hline T5 & 10 t ha-1 $^{-1}$ & Т18 & 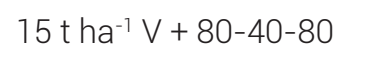 & Т31 & 5 t ha-1 $^{-}+120-60-120$ \\
\hline T6 & 15 t ha-1 $^{-1}$ & T19 & 5 t ha- $^{-1} V+40-20-40$ & T32 & 10 tha-1 $^{-1}+120-60-120$ \\
\hline T7 & 5 tha-1 $^{-}$ & T20 & 10 t ha-1 $^{-1}+40-20-40$ & Т33 & 15 t ha-1 $^{-} S+120-60-120$ \\
\hline T8 & $10 \mathrm{tha}^{-1} \mathrm{~S}$ & $\mathrm{~T} 21$ & 15 t ha-1 $^{-1} V+40-20-40$ & Т34 & $5 \mathrm{t} \mathrm{ha-1}^{-1} \mathrm{~S}+80-40-80$ \\
\hline T9 & $15 \mathrm{tha}^{-1} \mathrm{~S}$ & $\mathrm{~T} 22$ & 5 t ha-1 $^{-1}+120-60-120$ & T35 & $10 \mathrm{t} \mathrm{ha}^{-1} \mathrm{~S}+80-40-80$ \\
\hline Т10 & Fórmula 120-60-120 & $\mathrm{T} 23$ & 10 t ha-1 $^{-1}+120-60-120$ & Т36 & $15 \mathrm{tha}^{-1} \mathrm{~S}+80-40-80$ \\
\hline T11 & Fórmula 80-40-80 & $\mathrm{T} 24$ & 15 t ha-1 $^{-1}+120-60-120$ & T37 & 5 t ha-1 $^{-}+40-20-40$ \\
\hline T12 & Fórmula 40-20-40 & $\mathrm{T} 25$ & 5 t ha-1 $^{0}+80-40-80$ & T38 & $10 \mathrm{t} \mathrm{ha}^{-1} \mathrm{~S}+40-20-40$ \\
\hline T13 & 5 t ha-1 $^{-1}+120-60-120$ & T26 & 10 t ha $^{-1} 0+80-40-80$ & Т39 & $15 \mathrm{t} \mathrm{ha}^{-1} \mathrm{~S}+40-20-40$ \\
\hline
\end{tabular}

V: estiércol vacuno, O: estiércol ovino, S: Solep ${ }^{\oplus}$. 
Cuadro 2. Características fisicoquímicas de estiércoles y fertilizante orgánico comercial utilizados en el experimento de fuentes de abonos para la producción de fruto de chile poblano.

\begin{tabular}{lccccccccccc}
\hline Abono & $\mathrm{pH}$ & $\begin{array}{c}\mathrm{CE} \\
\left(\mathrm{dS} \mathrm{m} \mathrm{m}^{-1}\right)\end{array}$ & $\begin{array}{c}\text { Humedad } \\
(\%)\end{array}$ & $\begin{array}{c}\text { Cenizas } \\
(\%)\end{array}$ & $\begin{array}{c}\mathrm{MO} \\
(\%)\end{array}$ & $\begin{array}{c}\mathrm{CO} \\
(\%)\end{array}$ & $\begin{array}{c}\mathrm{N} \\
(\%)\end{array}$ & $\begin{array}{c}\mathrm{P} \\
(\%)\end{array}$ & $\begin{array}{c}\mathrm{K} \\
(\%)\end{array}$ & $\begin{array}{c}\mathrm{Ca} \\
(\%)\end{array}$ & $\begin{array}{c}\mathrm{Mg} \\
(\%)\end{array}$ \\
\hline Ovino & $8.96 \mathrm{a}$ & $7.18 \mathrm{a}$ & $0.07 \mathrm{c}$ & $44.09 \mathrm{c}$ & $8.36 \mathrm{a}$ & $4.85 \mathrm{a}$ & $13.30 \mathrm{a}$ & $0.26 \mathrm{a}$ & $0.57 \mathrm{a}$ & $2.85 \mathrm{a}$ & $10.24 \mathrm{a}$ \\
Vacuno & $8.37 \mathrm{~b}$ & $4.03 \mathrm{~b}$ & $0.09 \mathrm{~b}$ & $72.13 \mathrm{~b}$ & $3.33 \mathrm{~b}$ & $1.93 \mathrm{~b}$ & $7.70 \mathrm{~b}$ & $0.25 \mathrm{a}$ & $0.47 \mathrm{~b}$ & $0.93 \mathrm{c}$ & $1.98 \mathrm{~b}$ \\
Solep ${ }^{\circledR}$ & $7.97 \mathrm{~b}$ & $4.26 \mathrm{~b}$ & $0.10 \mathrm{a}$ & $77.30 \mathrm{a}$ & $2.83 \mathrm{~b}$ & $1.65 \mathrm{~b}$ & $6.33 \mathrm{c}$ & $0.23 \mathrm{~b}$ & $0.24 \mathrm{c}$ & $1.29 \mathrm{~b}$ & $1.89 \mathrm{~b}$ \\
DMS (0.05) & 0.500 & 0.275 & 0.008 & 1.380 & 0.824 & 0.478 & 0.083 & 0.009 & 0.010 & 0.316 & 0.244 \\
CV (\%) & 2.369 & 2.130 & 3.896 & 0.854 & 6.793 & 6.796 & 0.365 & 1.381 & 0.929 & 7.466 & 2.070 \\
Significancia & $* *$ & $* * *$ & $* * *$ & $* * *$ & $* * *$ & $* * *$ & $* * *$ & $* * *$ & $* * *$ & $* * *$ & $* * *$
\end{tabular}

Medias con letras iguales en la misma columna no son estadísticamente diferentes. CE: conductividad eléctrica, MO: materia orgánica, CO: carbono orgánico, N: nitrógeno, P. fósforo, K: potasio, Ca: calcio, Mg: magnesio, DMS: diferencia mínima significativa; CV: coeficiente de variación; ** y ***: significativas estadística a $\mathrm{P} \leq 0.01$ y $\mathrm{P} \leq 0.001$.

Cuadro 3. Cuadrados medios de los tratmientos (TR) y del error en el análisis de varianza del experimento de evaluación de fertilizantes orgánicos y químicos en chile poblano.

\begin{tabular}{|c|c|c|c|c|c|c|c|c|c|}
\hline Variable $^{\dagger}$ & TR & & Error & CV (\%) & Variable & TR & & Error & CV (\%) \\
\hline $\mathrm{DF}$ & 10.5 & ns & 8.1 & 6.4 & $\operatorname{LFr}(\mathrm{mm})$ & 79.9 & ns & 56.8 & 6.4 \\
\hline $\mathrm{DFr}$ & 28.0 & ns & 21.6 & 9.8 & $\operatorname{AFr}(\mathrm{mm})$ & 9.8 & ns & 9.2 & 4.9 \\
\hline AlP $(\mathrm{cm})$ & 46.9 & * & 20.5 & 6.7 & $\mathrm{GFr}(\mathrm{mm})$ & 11.9 & ns & 10.0 & 6.6 \\
\hline LTa (mm) & 9.5 & ns & 7.9 & 8.7 & $\mathrm{EPe}(\mathrm{mm})$ & 0.1 & ns & 0.1 & 8.4 \\
\hline DTa (mm) & 1.5 & ns & 1.3 & 10.2 & NLF & 0.1 & ns & 0.1 & 16.0 \\
\hline $\mathrm{NBi}$ & 69.9 & ns & 49.1 & 21.1 & NSF & 617.1 & ns & 950.0 & 12.9 \\
\hline $\mathrm{NFr}$ & 15.6 & * & 8.1 & 19.6 & PSe (g) & 0.5 & ns & 0.6 & 8.3 \\
\hline $\operatorname{PFr}(g)$ & 72.6 & * & 42.2 & 8.8 & $\operatorname{Ren}\left(\mathrm{t} \mathrm{ha}^{-1}\right)$ & 62.2 & * & 34.5 & 24.2 \\
\hline
\end{tabular}

†Para la descripción de las variables ver el apartado de variables respuesta en Materiales y Métodos. CV: coeficiente de variación; ns: no significancia; *: significancia a $\mathrm{P} \leq 0.05$.

comercial Solep ${ }^{\circledR}$ a 5 t ha-1 más fertilización química con la fórmula $80 \mathrm{~N}-40 \mathrm{P}-80 \mathrm{~K}$. Los resultados indican que al combinar estiércol con fertilización química se incrementa el rendimiento, al tener una sinergia entre las dos fuentes de nutrientes, a diferencia de la aplicación de cada una de las partes por separado.

El efecto positivo de la combinación de fuentes de nutrientes puede ser atribuible a que el estiércol de ovino presentó un alto contenido de materia orgánica, así como de los elementos N, P, K, Ca y Mg (Cuadro 2), los cuales son nutrientes esenciales para el desarrollo de las plantas (Li et al., 2016). Adicionalmente, la fertilización química, al suministrar N, P y K, mejora la nutrición del cultivo y aumenta la fertilidad del suelo con los nutrientes que aporta el estiércol de ovino; por lo tanto, la combinación de ambos genera una sinergia en la que están disponibles los elementos que necesita la planta para obtener sus nutrientes y aumentar el rendimiento.
Los resultados de esta investigación demuestran que es posible aumentar el rendimiento de fruto a $35.5 \mathrm{t} \mathrm{ha}^{-1}$, lo que es muy significativo ya que el rendimiento promedio de fruto fresco en la región de estudio fue de 8.4, 9.0,

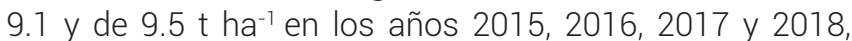
respectivamente (SIAP, 2019). Adicionalmente, la altura de planta y el número y peso de fruto también presentaron incrementos significativos, lo que puede ser atribuido al efecto positivo de combinar fertilizantes orgánicos y químicos, ya que el nitrógeno y otros nutrientes están disponibles durante mayor tiempo en las etapas de crecimiento y reproductiva.

El análisis de contrastes mostró que el estiércol vacuno, por sí solo, generó un rendimiento promedio de $21.5 \mathrm{t} \mathrm{ha}^{-1}$, mientras que con el estiércol ovino se obtuvo un rendimiento ligeramente inferior, de $19.2 \mathrm{t} \mathrm{ha}^{-1}$. La aplicación de los fertilizantes químicos por separado produjo un rendimiento promedio de $22.7 \mathrm{t} \mathrm{ha}^{-1}$. En términos de producción de fruto verde, los resultados muestran que cuando el efecto 
Cuadro 4. Comparación de medias de cuatro variables evaluadas en campo al aplicar fertilizantes orgánicos y fertilizantes químicos para la producción de chile poblano.

\begin{tabular}{|c|c|c|c|c|c|c|c|c|c|}
\hline Tratamientos & Descripción & Ren & t ha-1) & $\operatorname{AIP}($ & $(\mathrm{cm})$ & NF & & $\mathrm{PFr}$ & (g) \\
\hline 27 & 15 t ha- $^{-1} 0+80-40-80$ & 35.5 & a & 74.0 & a & 19 & a & 85.1 & a \\
\hline 26 & 10 t ha- $^{-1} 0+80-40-80$ & 34.0 & a & 72.0 & a & 17 & a & 86.5 & a \\
\hline 16 & 5 t ha-1 $^{-1}+80-40-80$ & 33.5 & a & 72.5 & a & 20 & a & 77.7 & a \\
\hline 20 & 10 t ha $^{-1} V+40-20-40$ & 31.0 & a & 71.5 & a & 17 & a & 81.0 & a \\
\hline 30 & 15 t ha-1 $^{-1}+40-20-40$ & 31.0 & a & 70.5 & a & 16 & a & 78.3 & a \\
\hline 31 & 5 t ha-1 $^{-1}+120-60-120$ & 31.0 & a & 75.5 & a & 17 & a & 74.1 & a \\
\hline 32 & 10 t ha-1 $^{-} S+120-60-120$ & 31.0 & a & 76.5 & a & 17 & a & 80.7 & a \\
\hline 15 & 15 t ha-1 $^{-1}+120-60-120$ & 30.5 & a & 71.0 & a & 16 & a & 79.1 & a \\
\hline 33 & 15 t ha-1 $^{-1}+120-60-120$ & 30.0 & a & 75.5 & a & 20 & a & 72.5 & \\
\hline 24 & 15 t ha-1 $^{-1} 0+120-60-120$ & 28.5 & a & 69.5 & a & 22 & a & 72.3 & \\
\hline 25 & 5 t ha-1 $^{0}+80-40-80$ & 28.0 & a & 70.5 & a & 16 & & 72.1 & \\
\hline 38 & 10 t ha-1 $^{-1}+40-20-40$ & 27.5 & a & 71.0 & a & 16 & & 76.6 & a \\
\hline 19 & 5 tha $^{-1} V+40-20-40$ & 27.0 & a & 65.5 & & 15 & & 75.8 & a \\
\hline 3 & 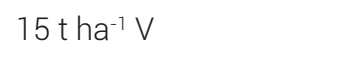 & 26.5 & a & 72.0 & a & 16 & & 73.1 & \\
\hline 8 & 10 tha $^{-1} \mathrm{~S}$ & 26.0 & a & 72.0 & a & 13 & & 82.9 & a \\
\hline 29 & 10 t ha-1 $^{-1}+40-20-40$ & 25.5 & a & 62.5 & & 14 & & 78.5 & a \\
\hline 39 & 15 t ha-1 $^{-1}+40-20-40$ & 25.5 & a & 69.0 & a & 13 & & 83.4 & a \\
\hline 6 & 15 t ha-1 $^{-1}$ & 25.0 & a & 68.5 & a & 15 & & 74.0 & a \\
\hline 10 & Fórmula 120-60-120 & 24.5 & a & 70.0 & a & 15 & & 66.8 & \\
\hline 23 & 10 t ha-1 $^{-1} 0+120-60-120$ & 24.0 & a & 68.0 & a & 17 & a & 69.6 & \\
\hline 12 & Fórmula 40-20-40 & 23.5 & & 69.0 & a & 15 & & 77.9 & a \\
\hline 14 & 10 t ha $^{-1} \mathrm{~V}+120-60-120$ & 23.5 & & 69.0 & a & 14 & & 71.5 & \\
\hline 18 & $15 \mathrm{tha}^{-1} \mathrm{~V}+80-40-80$ & 22.0 & & 70.5 & a & 13 & & 69.6 & \\
\hline 21 & 15 t ha $^{-1} V+40-20-40$ & 22.0 & & 62.5 & & 15 & & 70.1 & \\
\hline 28 & 5 t ha $^{-1} 0+40-20-40$ & 21.5 & & 60.5 & & 16 & & 73.8 & a \\
\hline 1 & $5 \mathrm{tha}^{-1} \mathrm{~V}$ & 20.5 & & 70.0 & a & 14 & & 70.1 & \\
\hline 22 & 5 t ha $^{-1} \mathrm{O}+120-60-120$ & 20.5 & & 63.5 & & 14 & & 66.4 & \\
\hline 11 & Fórmula 80-40-80 & 20.0 & & 70.5 & a & 12 & & 75.7 & a \\
\hline 36 & 15 t ha- $^{-1} S+80-40-80$ & 20.0 & & 69.5 & a & 13 & & 72.2 & \\
\hline 7 & 5 tha-1 $^{-} \mathrm{S}$ & 19.5 & & 55.5 & & 13 & & 75.6 & a \\
\hline 9 & $15 \mathrm{tha}^{-1} \mathrm{~S}$ & 19.0 & & 68.0 & a & 14 & & 65.7 & \\
\hline 13 & 5 t ha-1 $^{-1}+120-60-120$ & 19.0 & & 64.0 & & 13 & & 73.2 & \\
\hline 35 & 10 t ha-1 $^{-} S+80-40-80$ & 19.0 & & 66.0 & & 13 & & 70.0 & \\
\hline 5 & 10 tha $^{-1} \mathrm{O}$ & 18.0 & & 62.0 & & 11 & & 66.3 & \\
\hline 37 & $5 \mathrm{tha}^{-1} S+40-20-40$ & 18.0 & & 66.0 & & 12 & & 63.3 & \\
\hline 2 & $10 \mathrm{tha}^{-1} \mathrm{~V}$ & 17.5 & & 63.5 & & 11 & & 70.5 & \\
\hline 17 & 10 t ha- $^{-1} V+80-40-80$ & 17.5 & & 65.5 & & 13 & & 61.7 & \\
\hline 4 & 5 tha $^{-1} \mathrm{O}$ & 14.5 & & 60.0 & & 9 & & 68.0 & \\
\hline \multirow[t]{2}{*}{34} & $5 \mathrm{tha}^{-1} S+80-40-80$ & 14.0 & & 59.0 & & 9 & & 65.1 & \\
\hline & DMS (0.05) & 11.9 & & 9.2 & & 5.8 & & 13.2 & \\
\hline
\end{tabular}

Medias con letras iguales en la misma columna no son estadísticamente diferentes. Ren: rendimiento en fresco, AlP. altura de planta, NFr: número de frutos, PFr: peso de fruto, O: estiércol ovino, V: estiércol vacuno, S: Solep ${ }^{\circledR}$, DMS: diferencia mínima significativa. 
de ambas fuentes de elementos nutritivos se analiza por separado se presentan rendimientos promedio de fruto relativamente bajos; sin embargo, al combinar el estiércol con los fertilizantes químicos se aumenta el rendimiento, como también lo indican Ma et al. (2018) en trigo y Sileshi et al. (2011) en el cultivo de chile.

Con respecto al rendimiento del producto de interés comercial, Pandey et al. (2015) y Selvakumar et al. (2018) demostraron que la integración de estiércol con fertilizantes químicos representa una estrategia de manejo de nutrientes al favorecer una alta productividad y rendimiento en Tagetes minuta y en chile rojo, respectivamente. Esto ocurre porque el estiércol, al contener materia orgánica de lenta liberación de nutrientes que estarán disponibles por un periodo más largo, se puede complementar con la aplicación de fórmulas de fertilizantes químicos, ya que éstos son de liberación más rápida al contacto con el suelo y brindarán de inmediato los nutrientes que serán absorbidos por la raíz de la planta (Olowokere y TijaniEniola, 2013; Ribeiro et al., 2000).

El nitrógeno, presente en mayores cantidades en el estiércol ovino, lo mismo que otros elementos mayores (Cuadro 2), es necesario para la formación de proteínas y otras moléculas celulares, lo que da como resultado un incremento en el rendimiento de fruto al mejorar la nutrición en las plantas; el fósforo fue principalmente aportado por la fertilización química, ya que, tanto los estiércoles locales como el abono orgánico comercial, fueron muy similares entre ellos y con bajo contenido, éste tiene la función de mejorar el desarrollo de la raíz de la planta, así como la formación de frutos; el potasio, presente en mayor cantidad en el estiércol ovino, es importante durante el crecimiento de las plantas, ya que activa enzimas y desempeña un papel en el equilibrio del agua en la planta, lo que contribuye a mejorar el rendimiento y la calidad de los frutos (Chalkoo et al., 2014; Houdusse et al., 2007; Liu et al., 2014).

\section{CONCLUSIONES}

La combinación de estiércol ovino en nivel alto (15 t ha-1) en combinación con un nivel medio de fertilización química (80N-40P-80K) produjo los mayores rendimientos de fruto fresco por unidad de superficie en el cultivo de chile Poblano. La altura de planta, el número de frutos y el peso de fruto por planta fueron influenciadas positivamente por la aplicación combinada de ambas fuentes de nutrientes.

\section{BIBLIOGRAFÍA}

Acevedo-Alcalá P., J. Cruz-Hernández y 0. R. Taboada-Gaytán (2020) Abonos orgánicos comerciales, estiércoles locales y fertilización química en la producción de plántula de chile poblano. Revista Fitotecnia Mexicana 43:35-44, https://doi.org/10.35196/rfm.2020.1.35
Aliyu L. (2000) Effect of organic and mineral fertilizers on growth, yield and composition of pepper (Capsicum annuum L.). Biological Agriculture \& Horticulture 18:29-36, https://doi.org/10.1080/01448765.2000.9754862

Álvarez-Hernández J. C., S. Venegas-Flores, C. Soto-Ayala, A. ChávezVargas y L. Zavala-Sánchez (2011) Uso de fertilizantes químicos y orgánicos en cebolla (Allium cepa L.) en Apatzingán Michoacán, México. Avances en Investigación Agropecuaria 15:29-43.

Bindra A. D., P. Chopra y H. Lal (2014) Yield sustainability and chemical fertilizer economy through IPNS in capsicum (Capsicum annum L. var. grossum) under dry temperate condition of Himachal Pradesh. International Journal of Agriculture, Environment and Biotechnology 8:183-188, https://doi.org/10.5958/2230-732X.2015.00024.8

Bvenura C. and A. J. Afolayan (2013) Growth and physiological response to organic and/or inorganic fertilisers of wild Solanum nigrum L. cultivated under field conditions in Eastern Cape Province, South Africa. Acta Agriculturae Scandinavica Section B - Soil \& Plant Science 63:683-693, https://doi.org/10.1080/09064710.2013.852616

Chalkoo S., S. Sahay, A. Inam and S. Iqbal (2014) Application of wastewater irrigation on growth and yield of chilli under nitrogen and phosphorus fertilization. Journal of Plant Nutrition 37:11391147, https://doi.org/10.1080/01904167.2014.881864

Chami Z. A., I. Cavoski, D. Mondelli and T. Miano (2013) Effect of compost and manure amendments on zinc soil speciation, plant content, and translocation in an artificially contaminated soil. Environmental Science and Pollution Research International 20:4766-4776, https://doi.org/: 10.1007/s11356-012-1439-2

FAOSTAT, Organización de las Naciones Unidas para la Alimentación y la Agricultura, División de Estadística (2017) Rendimientos de chiles verdes y secos a nivel mundial año 2017. Organización de las Naciones Unidas para la Alimentación y la Agricultura. Roma. http://faostat.fao.org/beta/es/\#data/QC (Diciembre 2019).

Ghazali M. R., S. A. Mutalib and A. Abdullah (2016) Effect of cow manure and empty fruit bunches application treated with different fertilizers on growth and yield of chili (Capsicum annum). In: Proceedings of the 2016 UKM FST Postgraduate Colloquium. K. Ibrahim, K. H. Badri, M. H. Jumali, M. S. Noorani, N. Ibrahim, N. H. Rasol and W. A. W. Yaacob (eds.). University Kebangsaan Malaysia. AIP Publishing. Selangor, Malaysia. pp:1784, https://doi.org/10.1063/1.4966780

Gupta S., R. Kaushal, R. S. Spehia, S. S. Pathania and V. Sharma (2017) Productivity of capsicum influenced by conjoint application of isolated indigenous PGPR and chemical fertilizers. Journal of Plant Nutrition 40:921-927, https://doi.org/10.1080/01904167.2015.1093139

Houdusse F., M. Garnica and J. M. García-Mina (2007) Nitrogen fertiliser source effects on the growth and mineral nutrition of pepper (Capsicum annuum L.) and wheat (Triticum aestivum L.). Journal of the Science of Food and Agriculture 87:2099-2105, https://doi.org/10.1002/jsfa.2970

INEGI, Instituto Nacional de Estadística, Geografía e Informática (2009) Prontuario de información geográfica municipal de los Estados Unidos Mexicanos. Juan C. Bonilla, Puebla. http:// www3.inegi.org.mx/contenidos/app/mexicocifras/datos_ geograficos/21/21090.pdf (Septiembre 2020).

Kamaei R., F. Faramarzi, M. Parsa and M. Jahan (2019) The effects of biological, chemical, and organic fertilizers application on root growth features and grain yield of Sorghum. Journal of Plant Nutrition 42:2221-2233, https://doi.org/10.1080/01904167.2019.1648667

Khandaker M. M., F. Rohani, T. Dalorima and N. Mat (2017) Effects of different organic fertilizers on growth, yield and quality of Capsicum annuum L. Var. Kulai (Red Chilli Kulai). Biosciences Biotechnology Research Asia 14:185-192, https://doi.org/10.13005/bbra/2434

Li X., R. Zeng and H. Liao (2016) Improving crop nutrient efficiency through root architecture modifications. Journal of Integrative Plant Biology 58:193-202, https://doi.org/10.1111/jipb.12434

Liu R., Y. Kang, C. Zhang, L. Pei, S. Wan, S. Jiang, ... and Y. Yang (2014) Chemical fertilizer pollution control using drip fertigation for conservation of water quality in Danjiangkou Reservoir. Nutrient Cycling in Agroecosystems 98:295-307, https://doi. org/10.1007/s10705-014-9612-2

López A. M., J. E. Poot M. y M. A. Mijangos C. (2012) Respuesta del chile 
habanero (Capsicum chinense L. Jacq) al suministro de abono orgánico en Tabasco, México. Revista Científica UDO Agrícola 12:307-312

Ma C., Y. N. Liu, L. Liang, B. N. Zhai, H. Q. Zhang and Z. H. Wang (2018) Effects of combined application of chemical fertilizer and organic manure on wheat yield and leaching of residual nitrate- $\mathrm{N}$ in dryland soil (In Chinese). Ying Yong Sheng Tai Xue Bao 29:12401248, https://doi.org/10.13287/j.1001-9332.201804.023

Macías D. R., R. L. Grijalva C. y F. Robles C. (2012) Respuesta de la aplicación de estiércol y fertilizantes sobre el rendimiento y calidad del chile jalapeño. Biotecnia 14:32-38.

Moharana P. C., B. M. Sharma and D. R. Biswas (2017) Changes in the soil properties and availability of micronutrients after six-year application of organic and chemical fertilizers using STCRbased targeted yield equations under pearl millet-wheat cropping system. Journal of Plant Nutrition 40:165-176, https://doi.org/10.1080/01904167.2016.1201504

Montaño M. N. J., J. A. Simosa M. y A. J. Perdomo G. (2009) Respuesta de tres cultivares de berenjena (Solanum melogena L.) a diferentes combinaciones de fertilizante orgánico y fertilizante químico. Revista Científica UDO Agrícola 9:807-815.

Okazaki K., T. Shinano, N. Oka and M. Takebe (2010) Metabolite profiling of Raphanus sativus L. to evaluate the effects of manure amendment. Soil Science and Plant Nutrition 56:591-600 https://doi.org/10.1111/j.1747-0765.2010.00490.x

Olowokere F. A. and H. Tijani-Eniola (2013) Pepper response to inorganic and organomineral fertilizers in Southwestern Nigeria Communications in Soil Science and Plant Analysis 44:11271139, https://doi.org/10.1080/00103624.2012.750331

Pandey V., A. Patel and D. D. Patra (2015) Amelioration of mineral nutrition, productivity, antioxidant activity and aroma profile in marigold (Tagetes minuta L.) with organic and chemical fertilization. Industrial Crops and Products 76:378-385, https://doi.org/10.1016/j.indcrop.2015.07.023

Ribeiro L. G., J. C. Lopes, S. Martins Filho e S. S. Ramalho (2000) Adubação orgânica na produção de pimentão. Horticultura Brasileira 18:134 137, https://doi.org/10.1590/S0102-05362000000200012

Rodríguez A. E. A., M. M. Bolaños B. y J. C. Menjivar F. (2010) Efecto de la fertilización en la nutrición y rendimiento de ají (Capsicum spp.) en el Valle del Cauca, Colombia. Acta Agronómica 59:55-64

SAS Institute (2013) SAS/STAT ${ }^{\circledR} 9.3$ User's Guide. Statistical Analysis System Institute. Cary, North Carolina, USA. 8621 p.

Secretaría de Economía (2008) Norma Oficial Mexicana NMX-FF109-SCFI-2008, Humus de Lombriz (lombricomposta) Especificaciones y Métodos de Prueba. Secretaria de Economía México, D. F. http://www.economia-nmx.gob.mx/normas/ nmx/2007/nmx-ff-109-scfi-2008.pdf (Agosto 2020).

Selvakumar G., P. H. Yi, S. E. Lee and S. G. Han (2018) Influence of organic and inorganic fertilizer application on red pepper yield, soil chemical properties, and soil enzyme activities. Horticultural Science and Technology 36:789798, https://doi.org/10.12972/kjhst. 20180077

SIAP, Servicio de Información Agroalimentaria y Pesquera (2019) Anuario estadístico de la producción agrícola. Secretaría de Agricultura y Desarrollo Rural. Ciudad de México. https://nube.siap.gob. $\mathrm{mx} /$ cierreagricola/ (Diciembre 2019).

Sileshi G.W., F. K. Akinnifesi, F. M. Gondwe, 0. C. Ajayi, S. Mng'omba and K. Mwafongo (2011) Effect of organic fertilizer on the growth and fruit yield of six paprika (Capsicum annum L.) cultivars in Malawi. Agroforestry Systems 83:361-372, https://doi.org/10.1007/s10457-011-9415-2

Zeidan M. S. (2007) Effect of organic manure and phosphorus fertilizers on growth, yield and quality of lentil plants in sandy soil. Research Journal of Agriculture and Biological Sciences 3:748752 
